\title{
The Impact of Appendectomy in Clostridium difficile Infection and Length of Hospital Stay
}

Rajesh Essrani ${ }^{1,2}$, Dany Saturno ${ }^{3}$, Shehriyar Mehershahi ${ }^{4}$, Rajesh Kumar Essrani ${ }^{5}$, Muhammad Rajib Hossain $^{6}$, Shri Jai Kirshan Ravi ${ }^{7}$, Andrea Berger ${ }^{8}$, Asif Mehmood 1, 9

1. Internal Medicine, Geisinger Medical Center, Danville, USA 2. Internal Medicine, Lehigh Valley Health Network, Allentown, USA 3. General Internal Medicine, BronxCare Health System, Bronx, USA 4. Gastroenterology, BronxCare Health System, Bronx, USA 5. Internal Medicine, Bronx Lebanon Hospital Center, Bronx, USA 6. Hospital Medicine, Geisinger Medical Center, Danville, USA 7. General Internal Medicine, Guthrie Robert Packer Hospital, Sayre, USA 8. Biostatistics, Geisinger Medical Center, Danville, USA 9. Internal Medicine, Abington Hospital - Jefferson Health, Abington, USA

Corresponding author: Rajesh Essrani, raj1_essrani@hotmail.com

\section{Abstract}

\section{Introduction}

We aim to investigate Clostridium difficile infection (CDI) recurrence, severity, complications, and length of hospital stay in patients with and without prior history of appendectomy who were admitted to the hospital with CDI.

\section{Method}

We analyzed retrospective data for 862 patients, 18 years and older, with C. difficile inpatients diagnosed between January 1, 2017 and December 31, 2018 and sorted into two groups, with or without prior appendicectomy, to look for outcomes such as recurrence, hospital stay, complications, and related death in each group and use statistical analysis for comparison.

\section{Result}

There were 862 patients admitted with CDI, of which 122 (14.2\%) had a prior history of appendectomy and 740 (85.8\%) did not. Patients with an appendectomy prior were older (median age of $75 \mathrm{vs.} 69, \mathrm{p}=0.0033$ ) and had a higher proportion of females $(68.9 \%$ vs. $53.6 \%, \mathrm{p}=0.0017)$.

C. difficile recurrence in prior appendicectomy group vs. no appendectomy group was $12.3 \%$ and $9.3 \%$, respectively, but no statistical difference was noted $(\mathrm{p}=0.28)$. Also, there was no statistical difference in complications like ileus, colectomy, and mortality related to CDI in both groups. However, patients with appendectomies had significantly shorter hospital stays during C. difficile admission compared to patients without appendectomies (median of six days vs. seven days, $\mathrm{p}=0.0014$ ).

Received 07/09/2020

Review began $07 / 25 / 2020$ Review ended 08/05/2020 Published 09/09/2020

\section{๑) Copyright 2020}

Essrani et al. This is an open access article distributed under the terms of the Creative Commons Attribution License CC-BY 4.0., which permits unrestricted use, distribution, and reproduction in any medium, provided the original author and source are credited.

\section{Conclusion}

Our study shows that there is no statistical difference in the recurrence, severity, and complications of CDI in the presence or absence of the appendix but remarkably noted that people with prior appendicectomy had a shorter hospital stay.

Categories: Internal Medicine, Medical Education, Gastroenterology

Keywords: clostridium difficile, clostridium difficile infection, abdominal pain, antibiotics

\section{Introduction}

Clostridium difficile infection (CDI) is one of the most common infectious etiology of hospital-acquired (nosocomial) infections in hospitalized patients [1]. The generally accepted theory for the pathogenesis of C. difficile involves the disruption of normal intestinal flora, typically in the setting of prior antibiotic use, which allows C. difficile to proliferate [2]. The clinical spectrum of CDI ranges from mild diarrhea to fulminant colitis with shock, ileus, toxic megacolon, and death [3,4]. Recurrent CDI is defined by the resolution of CDI symptoms while on appropriate therapy, followed by the reappearance of symptoms within two to eight weeks after treatment has been stopped [1]. There are several risk factors for CDI and recurrence, such as age greater than 65 years, prolonged hospital stays, use of antibiotics other than C. difficile therapy, use of antacid, or immunosuppressive medications [5-7]. These risk factors suggest that the interaction between the host immunity and colonic flora disruption plays a significant role in CDI severity, recurrence, and length of stay.

The vermiform appendix is theorized to serve as a microbial reservoir and plays a vital role in the 
maintenance of colonic bacteria after serious gut infections $[8,9]$. The idea that the appendix has the ideal environment to maintain enteric bacteria in biofilms is reasonable, and its role in immune protection may be based upon providing normal colonic flora in times of need $[8,9]$.

We aim to investigate CDI recurrence, severity, complications, and length of hospital stay in patients with and without prior history of appendectomy who were admitted to the hospital with CDIs.

This article was presented as a poster presented at Digestive Disease Week, Chicago, IL, USA (Essrani, R. Mehershahi, S. Hossain, MR. Ravi, SJK, Berger, A. Mehmood, A. The Impact of Appendectomy in Clostridium Difficile Infection and Length of Hospital Stay; May 2020).

\section{Materials And Methods}

We analyzed retrospective data for 862 patients, 18 years and older, with C. difficile inpatients diagnosed between January 1, 2017 and December 31, 2018. The study was approved by the Institutional Review Board. The requirement of informed consent was waived at the time of approval due to the retrospective design. The charts of 87 patients who had been identified with a reoccurrence of $\mathrm{C}$. difficile after their index admission were manually reviewed by the investigator to confirm the reoccurrence and to assess if a patient was treated with a fecal microbiota transplant (FMT). Baseline characteristics were compared using Student's t-tests, Wilcoxon rank-sum tests, or Pearson's chi-square and Fisher's exact tests. Mortality was compared using Kaplan-Meier curves and log-rank tests. Due to high post-discharge patient mortality complications of C. difficile and reoccurrence were analyzed using Fine-Gray subdistribution hazard models where death was treated as a competing risk. Linear regression models with a natural log transformation of hospital length of stay were used to estimate the percentage decrease in stay days for patients with a prior appendectomy compared to those without. Results are also reported from a multivariable model adjusted for age and sex.

\section{Results}

There were 862 patients admitted with CDIs, of which 122 (14.2\%) had a prior history of appendectomy and 740 (85.8\%) did not. The median age at the time of admission was 70 years and $55.8 \%$ were female. Patients with an appendectomy prior were older (median age of 75 years vs. $69, \mathrm{p}=0.0033$ ) and had a higher proportion of females $(68.9 \%$ vs. $53.6 \%, p=0.0017)$. Groups were similar with respect to body mass index (BMI), lab values, blood pressure, and comorbid conditions (Table 1). 


\section{Cureus}

\begin{tabular}{|c|c|c|c|c|}
\hline & All Patients & Appendectomy Prior & No Appendectomy & P-Value \\
\hline Number of patients, $n$ & 862 & 122 & 740 & \\
\hline Age at C. difficile diagnosis, median (IQR) & $70(59,80)$ & $75(63,84)$ & $69(58,79)$ & 0.0033 \\
\hline BMI, median (IQR), n missing = 13 & $26.8(22.7,32.4)$ & $26.9(23.2,32.8)$ & $26.8(22.6,32.4)$ & 0.5620 \\
\hline WBC, median (IQR), n missing = 2 & $11.5(7.7,16.4)$ & $10.7(7.0,15.4)$ & $11.6(7.8,16.5)$ & 0.1073 \\
\hline Creatinine, median (IQR), $\mathrm{n}$ missing = 2 & $1.1(0.8,1.8)$ & $1.2(0.9,1.9)$ & $1.1(0.8,1.8)$ & 0.2593 \\
\hline Systolic blood pressure, mean (SD) & $125.0(26.5)$ & $126.8(26.3)$ & $124.7(26.5)$ & 0.4323 \\
\hline Diastolic blood pressure, mean (SD) & $70.3(17.7)$ & $70.2(15.3)$ & $70.3(18.1)$ & 0.9418 \\
\hline Sex, n (\%) & & & & 0.0017 \\
\hline Female & $481(55.8 \%)$ & $84(68.9 \%)$ & $397(53.6 \%)$ & \\
\hline Male & $381(44.2 \%)$ & $38(31.1 \%)$ & $343(46.4 \%)$ & \\
\hline Years from appendectomy to C. difficile, median (IQR) & N/A & $8.6(3.8,14.3)$ & N/A & N/A \\
\hline Year of C. difficile diagnosis, $n(\%)$ & & & & 0.5811 \\
\hline 2017 & $209(24.2 \%)$ & $32(26.2 \%)$ & $177(23.9 \%)$ & \\
\hline 2018 & $653(75.8 \%)$ & $90(73.8 \%)$ & $563(76.1 \%)$ & \\
\hline History of diabetes, n (\%) & $337(39.1 \%)$ & $54(44.3 \%)$ & $283(38.2 \%)$ & 0.2068 \\
\hline History of malignancy, $n(\%)$ & $302(35.0 \%)$ & 48 (39.3\%) & $254(34.3 \%)$ & 0.2816 \\
\hline History of cirrhosis, $n$ (\% & 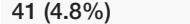 & $4(3.3 \%)$ & $37(5.0 \%)$ & 0.4079 \\
\hline
\end{tabular}

TABLE 1: Comparison of Baseline Demographic and Clinical Characteristics of Patients With and Without Prior History of Appendectomy

C. difficile, Clostridium difficile; IQR, interquartile range; BMI, body mass index; WBC, white blood cell; SD, standard deviation.

There was no statistical difference in complications like ileus, colectomy, and recurrence of CDI in both groups. A total of 33 patients (27.3\%) with a history of appendectomy died during the study period compared to 264 patients $(35.9 \%)$ without an appendectomy (log-rank p-value $=0.0736)$. Patients with appendectomies had significantly shorter hospital stays during C. difficile admission compared to those without appendectomies (median of six days vs. seven days, $\mathrm{p}=0.0014$ ) (Table 2). 


\section{Cureus}

\begin{tabular}{|c|c|c|c|c|}
\hline & All Patients & Appendectomy Prior & No Appendectomy & P-Value \\
\hline Number of patients, $\mathrm{n}$ & 862 & 122 & 740 & \\
\hline lleus, $\mathrm{n}(\%)$ & $84(9.7 \%)$ & $14(11.5 \%)$ & $70(9.5 \%)$ & 0.5038 \\
\hline Megacolon, n (\%) & $0(0.0 \%)$ & $0(0.0 \%)$ & $0(0.0 \%)$ & N/A \\
\hline Colectomy, n (\%) & $16(1.9 \%)$ & $2(1.6 \%)$ & 14 (1.9\%) & 0.8388 \\
\hline Complication (illeus or colectomy), $\mathrm{n}(\%)$ & $92(10.7 \%)$ & $14(11.5 \%)$ & $78(10.5 \%)$ & 0.7831 \\
\hline Clostridium difficile reoccurrence, n (\%) & $84(9.8 \%)$ & 15 (12.3\%) & 69 (9.3\%) & 0.2805 \\
\hline Deceased, $n(\%), n$ missing = 5 & 297 (34.7\%) & $33(27.3 \%)$ & $264(35.9 \%)$ & 0.0736 \\
\hline Length of stay days, median (IQR) & $6(4,11)$ & $6(4,8)$ & $7(4,11)$ & 0.0014 \\
\hline
\end{tabular}

TABLE 2: Comparison of Outcomes for Patients With and Without Prior History of Appendectomy

IQR, interquartile range.

Patients with a prior appendectomy stayed $23.4 \%$ fewer days in the hospital compared to patients without appendectomies (95\% CI: $-33.4 \%,-9.6 \%)$. After adjusting for age and sex, patients who had a prior appendectomy stayed $23.3 \%$ fewer days in the hospital (95\% CI: $-34.3 \%,-10.6 \%$ ).

A total of 84 patients experienced a reoccurrence of C. difficile, of which 15 (17.9\%) had appendectomies prior and 69 (82.1\%) did not. One appendectomy patient and three non-appendectomy patients received an FMT. All four patients received an OpenBiome transplant. Patients did not differ with respect to baseline characteristics or if they received FMT (Table 3). 


\section{Cureus}

\begin{tabular}{|c|c|c|c|c|}
\hline & All Patients With Reoccurrence & Appendectomy Prior & No Appendectomy & P-Value \\
\hline Number of patients, $\mathrm{n}$ & 84 & 15 & 69 & \\
\hline Age at $\mathrm{C}$. difficile diagnosis, median (IQR) & $74(61,83)$ & $76(61,85)$ & $72(61,82)$ & 0.4200 \\
\hline BMI, median (IQR), n missing = 2 & $26.7(22.7,32.5)$ & $27.3(26.0,34.7)$ & $26.7(22.4,32.0)$ & 0.2569 \\
\hline WBC, median (IQR), n missing = 1 & $11.7(7.7,16.6)$ & $10.7(6.9,14.6)$ & $11.8(7.8,17.1)$ & 0.7107 \\
\hline Creatinine, median (IQR), n missing = 1 & $1.2(0.8,2.1)$ & $1.6(0.9,2.2)$ & $1.2(0.8,1.9)$ & 0.2447 \\
\hline Systolic blood pressure, mean (SD) & $125.7(23.4)$ & $130.3(23.1)$ & $124.7(23.5)$ & 0.4040 \\
\hline Diastolic blood pressure, mean (SD) & $68.1(17.4)$ & $65.6(19.1)$ & $68.7(17.1)$ & 0.5391 \\
\hline Sex, n (\%) & & & & 0.4701 \\
\hline Female & $49(58.3 \%)$ & $10(66.7 \%)$ & $39(56.5 \%)$ & \\
\hline Male & $35(41.7 \%)$ & $5(33.3 \%)$ & $30(43.5 \%)$ & \\
\hline Years from appendectomy to $\mathrm{C}$. difficile, median (IQR) & N/A & $5.9(2.0,15.4)$ & N/A & N/A \\
\hline Year of C. difficile diagnosis, n (\%) & & & & 0.7500 \\
\hline 2017 & $23(27.4 \%)$ & $3(20.0 \%)$ & $20(29.0 \%)$ & \\
\hline 2018 & $61(72.6 \%)$ & $12(80.0 \%)$ & $49(71.0 \%)$ & \\
\hline History of diabetes, $n$ (\%) & $45(53.6 \%)$ & $10(66.7 \%)$ & $35(50.7 \%)$ & 0.2618 \\
\hline History of malignancy, $\mathrm{n}(\%)$ & $37(44.0 \%)$ & $6(40.0 \%)$ & $31(44.9 \%)$ & 0.7275 \\
\hline History of cirrhosis, $n$ (\%) & $3(3.6 \%)$ & $1(6.7 \%)$ & $2(2.9 \%)$ & 0.4501 \\
\hline History of FMT, n (\%) & $4(4.8 \%)$ & $1(6.7 \%)$ & $3(4.3 \%)$ & 0.5520 \\
\hline OpenBiome FMT, n (\%) & $4(100.0 \%)$ & $1(100.0 \%)$ & $3(100.0 \%)$ & N/A \\
\hline Mode of FMT delivery, $\mathrm{n}(\%)$ & & & & 1.0000 \\
\hline Oral capsule & $0(0.0 \%)$ & $0(0.0 \%)$ & $0(0.0 \%)$ & \\
\hline Colonoscopy & $3(75.0 \%)$ & $1(100.0 \%)$ & $2(66.7 \%)$ & \\
\hline Upper endoscopy & $1(25.0 \%)$ & $0(0.0 \%)$ & $1(33.3 \%)$ & \\
\hline difficile reocc & $25.0 \%$ & $.0 \%$ & $3 \%$ & 1.0000 \\
\hline
\end{tabular}

TABLE 3: Comparison of Patients With and Without Prior History of Appendectomy Who Experienced Reoccurrence

C. difficile, Clostridium difficile; BMI, body mass index; IQR, interquartile range; WBC, white blood cell; SD, standard deviation; FMT, fecal microbiota transplant.

\section{Discussion}

C. difficile colitis is a highly prevalent infection cause by an anaerobic, gram-positive, spore-forming bacteria known as $\mathrm{C}$. difficile. The $\mathrm{CDI}$ has a wide spectrum of clinical presentation ranging from nonsevere, severe to a fulminant colitis [3,4,10]. An epidemiologic study conducted in 2011 identified 453,000 cases and 29,000 deaths associated with CDI [11].

It has been hypothesized that the appendix serves as a microbial reservoir and plays a vital role in the maintenance of colonic bacteria after serious gut infections; therefore, there is the impression that patients who underwent appendectomy are at a higher risk of developing recurrent infection but not initial onset of C. difficile-associated colitis [12].

Current data demonstrate conflicting evidence on repercussions of a prior appendectomy on CDI. According to Im et al., patients who had an appendectomy done were found to have an increased risk of recurrent CDI and were also associated with a more severe clinical course [13]. In contrast, Franko et al. found no effect of 
appendectomy status on recurrence of CDI [14]. The aim of our study was to determinate CDI recurrence, severity, complications, and length of hospital stay in patients with and without prior history of appendectomy who were admitted to the hospital with CDIs.

As mentioned in the results, among 862 patients who were included in the study, only 122 patients had an appendectomy done in the past, and no statistical difference in the incidence of recurrence was found between the two groups (15 patients with prior appendectomy who represent $12.3 \%$ of this subgroup and 69 patients without appendectomy who represent $9.3 \%$ of this subgroup had recurrent CDI). Older age, renal failure, and underlying comorbidities are frequent risk factors for complicated CDI [15]. In our subgroup with prior appendectomy, the median age was 75 years, which was higher compared to the non-appendectomy group that was 69 years. This could be a confounding factor that may have created a difference between the two groups, contraintuitive to what would be expected the group post appendectomy would be at a higher risk for recurrence (explained by their age and by appendectomy itself) compared to non-appendectomy group, but our results as mentioned before showed no difference. Data from multiple studies have suggested a possible protective role of the appendix.

The appendix has been described as a well-known site of production of IgA, the predominant immunoglobulin in the gut associated lymphoid tissue (GALT) system [16]. Several studies have pointed to an association between IgA levels and C. difficile colonization and infection [17]. According to Azrad et al., serum IgA was shown to block inflammatory response by suppression of phagocytosis, chemotaxis, and cytokine production [18]. Considering this information, it would be expected that the non-appendectomy patients would have a less severe form of this infection and therefore have a shorter length of stay compared to the appendectomy patients. In contrast, our study found that patients with appendectomies had significantly shorter hospital stays compared to patients without appendectomies. We can imply this difference could be related to faster response to treatment in the case of appendectomized patients. Because of the lack of good evidence on the subject, we suggest further study on the matter.

The study has several limitations as it was a retrospective study, with a relatively small sample size that reflects only those that came in our hospital from January 1, 2017 and December 31, 2018. Due this we were not able to do stratified analysis to rule out other confounding factors, such as difference on age, proton pump inhibitor (PPI) consumption, enteral feeding, and gastrointestinal surgery.

\section{Conclusions}

Despite what has been described in previous studies, we found there is no statistical difference in the recurrence, severity, and complications of CDI in the presence or absence of the appendix but remarkably noted that people with prior appendicectomy had a shorter hospital stay. Most of literature on this subject is based on observational data and retrospective studies that limits external validity of the data; further, prospective studies are warranted to validate the result.

\section{Additional Information \\ Disclosures}

Human subjects: Consent was obtained by all participants in this study. Geisinger Institutional Review Board issued approval 2019-0184. Your protocol was reviewed and it was determined that your research protocol meets the criteria for EXEMPTION. . Animal subjects: All authors have confirmed that this study did not involve animal subjects or tissue. Conflicts of interest: In compliance with the ICMJE uniform disclosure form, all authors declare the following: Payment/services info: All authors have declared that no financial support was received from any organization for the submitted work. Financial relationships: All authors have declared that they have no financial relationships at present or within the previous three years with any organizations that might have an interest in the submitted work. Other relationships: All authors have declared that there are no other relationships or activities that could appear to have influenced the submitted work.

\section{References}

1. McDonald LC, Gerding DN, Johnson S, et al.: Clinical practice guidelines for Clostridium difficile infection in adults and children: 2017 Update by the Infectious Diseases Society of America (IDSA) and Society for Healthcare Epidemiology of America (SHEA). Clin Infect Dis. 2018, 66:e1-e48.

2. Barbut F, Petit JC: Epidemiology of Clostridium difficile-associated infections. Clin Microbiol Infect. 2001, 7:405-410. 10.1046/i.1198-743x.2001.00289.x

3. Monaghan T, Boswell T, Mahida YR: Recent advances in Clostridium difficile-associated disease . Postgrad Med J. 2009, 85:152-162. 10.1136/gut.2007.128157

4. Fekety R, McFarland LV, Surawicz CM, Greenberg RN, Elmer GW, Mulligan ME: Recurrent Clostridium difficile diarrhea: characteristics of and risk factors for patients enrolled in a prospective, randomized, double-blinded trial. Clin Infect Dis. 1997, 24:324-333.

5. Aslam S, Musher DM: An update on diagnosis, treatment, and prevention of Clostridium difficile-associated disease. Gastroenterol Clin North Am. 2006, 35:315-335. 10.1016/j.gtc.2006.03.009

6. Hu MY, Katchar K, Kyne L, et al.: Prospective derivation and validation of a clinical prediction rule for recurrent Clostridium difficile infection. Gastroenterology. 2009, 136:1206-1214. 
7. T. Monaghan, T. Boswell, Y.R: Recent advances in Clostridium difficile-associated disease . Postgrad Med J. 85:152-162. 10.1136/gut.2007.128157

8. Randal Bollinger R, Barbas AS, Bush EL, Lin SS, Parker W: Biofilms in the large bowel suggest an apparent function of the human vermiform appendix. J Theor Biol. 2007, 249:826-831. 10.1016/j.jtbi.2007.08.032

9. Ansaloni L, Catena F, Pinna AD: What is the function of the human vermiform appendix?. Evolution-based surgery: a new perspective in the Darwinian year 2009. Eur Surg Res. 2009, 43:67-71.

10. Surawicz CM, Brandt LJ, Binion DG, et al.: Guidelines for diagnosis, treatment, and prevention of Clostridium difficile infections. Am J Gastroenterol. 2013, 108:478-498. 10.1038/ajg.2013.4

11. Lessa FC, Mu Y, Bamberg WM, et al.: Burden of Clostridium difficile infection in the United States . N Engl J Med. 2015, 372:825-834. 10.1056/NEJMoa1408913

12. Sanders NL, Bollinger RR, Lee R, Thomas S, Parker W: Appendectomy and Clostridium difficile colitis: relationships revealed by clinical observations and immunology. World J Gastroenterol. 2013, 19:5607-5614. 10.3748/wjg.v19.i34.5607

13. Im GY, Modayil RJ, Lin CT, Geier SJ, Katz DS, Feuerman M, Grendell JH: The appendix may protect against Clostridium difficile recurrence. Clin Gastroenterol Hepatol. 2011, 9:1072-1077. 10.1016/j.cgh.2011.06.006

14. Franko J, Ferrel B, Pierson P, et al.: Influence of prior appendectomy and cholecystectomy on Clostridioides difficile infection recurrence and mortality. Am J Surg. 2020, 220:203-207. 10.1016/j.amjsurg.2019.10.038

15. Abou Chakra CN, Pepin J, Sirard S, Valiquette L: Risk factors for recurrence, complications and mortality in Clostridium difficile infection: a systematic review. PLoS One. 2014, 9:e98400. 10.1371/journal.pone.0098400

16. Dasso JF, Obiakor H, Bach H, Anderson AO, Mage RG: A morphological and immunohistological study of the human and rabbit appendix for comparison with the avian bursa. Dev Comp Immunol. 2000, 24:794814. 10.1016/S0145-305X(00)00033-1

17. Bridgman SL, Konya T, Azad MB, et al.: High fecal IgA is associated with reduced Clostridium difficile colonization in infants. Microbes Infect. 2016, 18:543-549. 10.1016/j.micinf.2016.05.001

18. Azrad M, Hamo Z, Tkhawkho L, Peretz A: Elevated serum immunoglobulin A levels in patients with Clostridium difficile infection are associated with mortality. Pathog Dis. 2018, 76:fty066. 